\title{
The Kidney in Bardet-Biedl Syndrome: Possible Pathogenesis of Urine Concentrating Defect
}

\author{
Miriam Zacchia ${ }^{a}$ Valentina Di lorio $^{b}$ Francesco Trepiccione $^{a}$ \\ Marianna Caterino $^{c}$ Giovambattista Capasso $^{a}$ \\ ${ }^{a}$ Division of Nephrology, Department of Cardiothoracic and Respiratory Sciences, University of Campania \\ "Luigi Vanvitelli," b Eye Clinic, Multidisciplinary Department of Medical, Surgical, and Dental Sciences, \\ University of Campania "Luigi Vanvitelli," and 'Department of Molecular Biology and Medical Biotechnologies, \\ Università degli Studi di Napoli "Federico II," Naples, Italy
}

\section{Keywords}

Aquaporin 2 - Bardet-Biedl syndrome · Hyposthenuria . Uromodulin

\begin{abstract}
Background: The ciliopathies are a growing number of disorders caused by mutations in genes involved in the function of the primary cilium. Bardet-Biedl syndrome (BBS) belongs to this group of disorders. In this setting, kidney dysfunction is highly variable, and urine concentrating defect, a common feature of multiple ciliopathies, has been described as the most frequent defect. Here we review the mechanism of urine concentration and describe the possible mechanism underling this defect in ciliopathies and especially BBS, based on the current body of literature. Summary: Active $\mathrm{Na}^{+}$absorption along the thick ascending limb of the loop of Henle (TAL) is critical for generating the corticomedullary osmotic gradient, and the countercurrent anatomical arrangement of the 2 branches of the loop of Henle enhances this gradient. The vasa recta, paralleling the loop of Henle, operate into the countercurrent mechanism, minimizing washout of solutes from the interstitium. Final water reabsorption is mediated by the aquaporin 2 (AQP2) water channels along
\end{abstract}

\section{KARGER}

E-Mail karger@karger.com

www.karger.com/kdd the distal nephron, and it is under hormonal control. Several studies demonstrated that hyposthenuria in BBS patients relies on kidney resistance to desmopressin, suggesting a renal origin. We recently showed that the majority of hyposthenuric BBS patients have also a defect regarding maximal urine dilution. Independent studies showed that BBS10 deficiency caused AQP2 mistrafficking in vitro; accordingly, we demonstrated impaired urinary AQP2 excretion in BBS patients with combined concentrating and diluting defect. Whether receptor signaling pathways or downstream events cause AQP2 deregulation is still unclear. In addition, reduced urinary uromodulin excretion in BBS patients opens the possibility that TAL dysfunction may also play a pathogenic role. Key Message: Impaired water handling in BBS is associated with AQP2 mistrafficking. The potential role of additional factors, such as the dissipation of the medullary osmotic gradient due to TAL dysfunction and/or structural anomalies, remains to be elucidated.

(c) 2017 S. Karger AG, Basel

M. Zacchia and V. Di lorio contributed equally to this work. Contribution from the International Conference: The Kidney in Genetic and Rare Diseases, Naples, October 27-29, 2016. 


\section{Introduction}

Bardet-Biedl syndrome (BBS) is a rare multisystem disorder. Laurence and Moon described the first case in 1866; the main clinical features were obesity, retinal degeneration, paraparesis, and cognitive impairment [1]. Later, Bardet [2] and Biedl [3] described 2 similar clinical cases who showed, in addition, polydactyly and hypogenitalism, and the disease was named Laurence-Moon-Bardet-Biedl syndrome. Laurence-Moon syndrome and Bardet-Biedl syndrome have been considered 2 different diseases [4]; however, there are several overlapping features suggesting that they could be part of the same disease spectrum.

Clinically, BBS is highly variable. The main clinical features are retinal degeneration, polydactyly, obesity, learning disabilities, hypogenitalism, and renal anomalies. Secondary features include brachydactyly, syndactyly, craniofacial dysmorphism, diabetes mellitus, behavioral abnormalities, oro-dental anomalies, hepatic fibrosis, and Hirschsprung disease [4]. Generally, the spectrum of clinical manifestations is complete after the first decade of life, and the median age of patients at diagnosis is 9 years [4]. Therefore, the diagnosis is often missed at birth. The classical story of a BBS patient is characterized by an uneventful pregnancy, with normal body weight at birth. The most common congenital defect is polydactyly, which can be seen on ultrasound prenatally or be discovered at birth [5]. However, this finding is nonspecific, and without the other major signs it is hard to predict BBS. During infancy, obesity onset $[6,7]$ and developmental delays are frequent. Some patients show prenatal ultrasound evidence of renal and urinary tract malformation, such as cystic kidney, pelvic dilation, and ureter obstruction. The association of kidney abnormalities and polydactyly may alert the physician to the diagnosis. Symptoms of retinal dystrophy develop during the first decade of life and include either central (acuities) or more peripheral (threshold) functions, with progressive acuity decline [8].

The genetic heterogeneity may at least in part explain phenotypic variability. To date, 21 genes have been associated with human BBS [9]. The majority of the known BBS genes are involved in the function of the primary cilium. While motile cilium is required for fluid propulsion and cell motility, the primary cilium is nonmotile; conversely, it has a pivotal role in several signal transduction pathways [10]. The role of BBS proteins in ciliary function was highlighted by the discovery that mammalian BBS8 was restricted to ciliated cells [11]. Subsequent studies demonstrated that several other BBS proteins are implicated in the function of the primary cilium [12]. In recent years, many efforts have shed light onto the function of these proteins. It has been shown that $8 \mathrm{BBS}$ subunits (BBS1, BBS2, BBS4, BBS5, BBS7, BBS8, BBS9 and BBIP10/BBS18) form a multiprotein complex called BBSome. BBSome dysfunction strongly affects the protein composition of the ciliary membrane, suggesting its role in the trafficking of molecules to the cilium [13]. In addition, in Caenorhabditis elegans, the BBSome is also required to assemble the intraflagellar transport particles, a multiprotein complex mediating bidirectional movement of nonmembrane molecules along the axoneme and between the axoneme and the membrane [14]. The understanding of the function of the remaining BBS genes is increasing thanks to the development of animal models [15]. Several interacting subunits have been identified. The BBS1, BBS4, BBS5, and BBS8 subunits have been shown to interact with polycystin 1 , the protein mutated in autosomal dominant polycystic kidney disease (ADPKD), and BBS1 knockdown caused impaired polycystin 1 trafficking along the cilium in renal epithelial cells [16]. The BBSome has been shown to be required for the trafficking of multiple $G$ protein-coupled receptors, a large number of transmembrane proteins involved in cellular response to several sensory inputs [17], including the melanin-concentrating hormone receptor 1, and somatostatin receptor 3 , which causes blindness and obesity [18].

In humans, no clear phenotype-genotype correlation has been proved. Some reports showed that BBS1 mutations are associated with a less severe phenotype compared with other BBS genes [19], especially BBS10 [20, 21]. However, in a recent study, it was demonstrated that biallelic truncating mutations in any gene correlated with more severe renal disease compared with missense mutations, suggesting that any BBS gene, when strongly affected, may result in severe kidney dysfunction [21].

\section{The Kidney in BBS}

\section{Renal Structural and Functional Abnormalities}

The renal phenotype is highly variable in BBS. The earliest reports of BBS cases did not describe renal involvement. To our knowledge, the first descriptions of renal impairment in BBS individuals appeared during the 1980s [22-24]. Since then, several studies have reported, with a high frequency, renal impairment and urinary tract malformations, and kidney defects have been included among the cardinal features of the disease [4].
58

Kidney Dis 2017;3:57-65 DOI: $10.1159 / 000475500$
Zacchia/Di Iorio/Trepiccione/Caterino/ Capasso 
Multiple structural kidney anomalies have been described, including fetal lobulations, parenchymal cysts, calyceal cysts and clubbing, and renal agenesia [4]. Also, horseshoe kidney and urinary tract malformation (such as vesicoureteral reflux and urethral stenosis) have been described. In the study by Beales et al. [4] in 1999, 46\% of 57 patients had renal malformation; O’Dea et al. [25] described the presence of kidney structural abnormalities in $96 \%$ of 38 BBS patients. Accordingly, in our Italian cohort of 41 BBS patients, only 4 subjects did not show any kidney defect on ultrasound, providing further evidence of a high frequency of renal structural anomalies [7]. Several renal functional abnormalities have been described.

The rate of glomerular filtration rate (GFR) decline varies among the studies in the literature. Beales et al. [4] described presence of renal insufficiency (defined as a rise in plasma creatinine levels or requirement of renal replacement therapy) in $9 \%$ of 109 patients. O'Dea et al. [25] showed that among $36 \mathrm{BBS}$ patients, $25 \%$ had an estimated creatinine clearance $<70 \mathrm{~mL} / \mathrm{min}$. In a recent retrospective study conducted in $350 \mathrm{BBS}$ individuals, it was shown that $45 \%$ of adult and $46 \%$ of pediatric BBS patients had chronic kidney disease (CKD) stage I-V, suggesting a high rate of kidney dysfunction in BBS subjects [21]. However, severe renal impairment occurred in a small fraction of patients, and 6 and $8 \%$ of pediatric and adult subjects were in CKD stage IV-V. The low incidence of patients with latest stages of CKD in this report may be explained in 2 manners: (1) BBS patients may have slow progression of kidney disease, and (2) BBS patients with CKD, even at early stages, have a high risk of adverse outcomes, a reasonable possibility, in common with CKD patients for any cause.

In accordance with data from the literature, in our cohort of 41 Italian BBS patients, we found a high incidence of structural abnormalities and renal dysfunction, with $12 \%$ of patients in CKD stage III-V [7]. Since BBS patients with advanced CKD are a small fraction of an already rare disease, it is hard to predict renal outcome in this setting. According to our observations, it seems that BBS patients with severe kidney dysfunction may be divided into 2 groups, the first one including patients with a story of renal and urinary tract abnormalities diagnosed early in life (including antenatal cystic disease and urogenital sinus persistence), leading to the end-stage renal disease in childhood/adolescence, and the second one including adult BBS patients with no clear information on the onset of CKD.

Whether adult BBS patients have a higher risk of developing $\mathrm{CKD}$ than the general population is unknown.

The Kidney in Bardet-Biedl Syndrome
Given the rarity of the disease and the lack of longitudinal studies, it is hard to define the risk factors for kidney disease progression. There is no doubt that among BBS individuals there is a high incidence of obesity and diabetes mellitus; in addition, it is common to find urological defects; it is possible that those factors may favor the development of CKD. Whether BBS-specific untypical risk factors may favor renal disease remains to be demonstrated.

Occurrence of tubular dysfunction is not rare; some patients have been described to show renal tubular acidosis [26]. However, the most common kidney dysfunction is urine concentrating defect due to renal resistance to vasopressin/desmopressin [26]. Thus, the renal phenotype in BBS patients shows a wide range of severity, from GFR decline to tubular dysfunction in the absence of renal insufficiency. The reason for this variability is due, at least in part, to the high genetic heterogeneity. However, the rarity of the disease illustrated the difficulties in accurately assessing a genotype-phenotype correlation. The molecular mechanism underlying hyposthenuria is currently largely unknown; in addition, whether this defect may represent a presenting symptom preceding a progressive GFR decline is unknown at present.

\section{Pathophysiology of Urine Concentration and Dilution}

Renal water balance is maintained by finely tuning urine concentration and dilution. In water restriction conditions, to avoid a life-threatening increase in plasma osmolality, water reabsorption from urine is maximized, determining an increased urine osmolality (uOsm) of up to $1,200 \mathrm{mOsm} / \mathrm{kg} \mathrm{H}_{2} \mathrm{O}$ in humans; conversely, during high water intake, plasma osmolality is kept in the physiological range by increased free water clearance, leading to the production of dilute urine with an expected $\mathrm{uOsm}<100 \mathrm{mOsm} / \mathrm{kg} \mathrm{H}_{2} \mathrm{O}$. As total urine solute excretion rate varies a little between these conditions, the readout of these changes is the variation of water reabsorption [27].

This broad variation of $\mathrm{uOsm}$ is determined by $2 \mathrm{fac}-$ tors: (1) the generation of the medullary osmotic gradient and (2) the possibility to modulate the water permeability of the collecting duct (CD) according to body water content.

The generation of the interstitial corticomedullary osmotic gradient is the result of a complex functional interaction among active solute transport, selective water permeability along specific nephron segments, and the countercurrent configuration of the medullary nephron sites and vasa recta (Fig. 1).

Kidney Dis 2017;3:57-65 DOI: $10.1159 / 000475500$ 


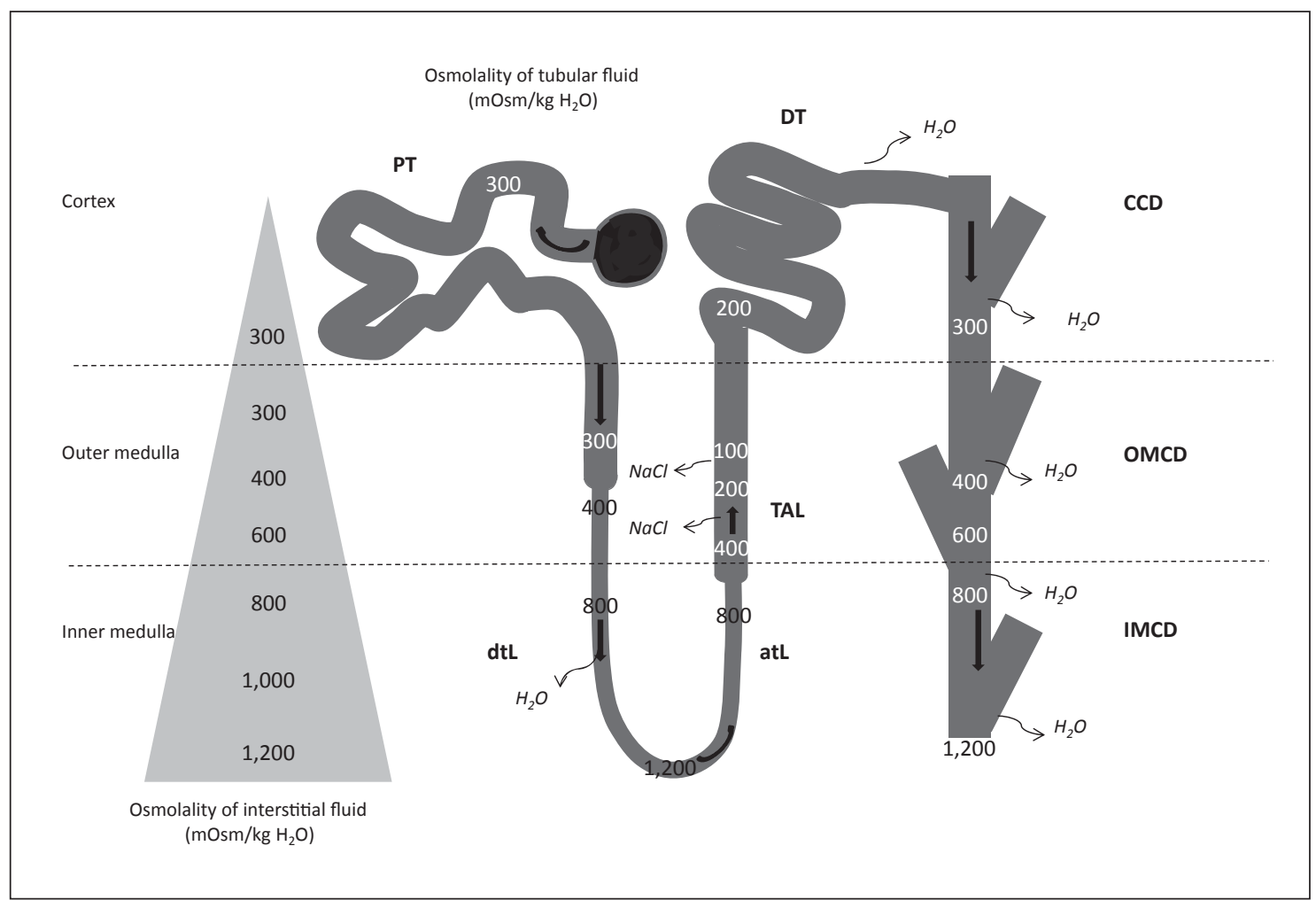

Fig. 1. Schematic representation of the nephron segments involved in urine concentration and dilution. atL, ascending thin limb of the loop of Henle; CCD, cortical collecting duct; DT, distal tubule; dtL, descending thin limb of the loop of Henle; IMCD, inner medullary collecting duct; OMCD, outer medullary collecting duct; PT, proximal tubule; TAL, thick ascending limb of the loop of Henle.

A water-independent active $\mathrm{Na}^{+}$absorption along the thick ascending limb of the loop of Henle (TAL) on the outer medulla is critical for both diluting luminal fluid and accumulating electrolytes in the interstitium, leading to an osmotic disequilibrium between the lumen and the interstitium [28]. This mechanism is mediated by the furosemide-sensitive $\mathrm{Na}-\mathrm{K}-2 \mathrm{Cl}$ cotransporter (NKCC2). Blocking the NKCC2 results in both urine concentrating and diluting defects, leading to iso-osmotic urine. Accordingly, hereditary disorders affecting salt absorption along the TAL, as in Bartter syndrome, result in a urine concentrating defect and are classified as secondary inherited nephrogenic diabetes insipidus [29]. The osmotic gradient generated by active $\mathrm{NaCl}$ absorption along the TAL is enhanced by the countercurrent anatomical arrangement of the 2 branches of the loop of Henle, rising from the corticomedullary junction to the outer-inner medullary boundary, in a process defined as countercurrent multiplication [30]. The vasa recta, paralleling the loop of Henle, operate into the countercurrent mecha- nism, minimizing washout of solutes from the interstitium.

The descending limb of the loop of Henle (dtL) is highly water-permeable due to the constitutive expression of aquaporin 1 (AQP1) at both the apical and the basolateral plasma membrane. Thus, the fluid entering the $\mathrm{dtL}$ is continuously in equilibrium with the interstitium, with a progressive increased osmolality from the outer medulla to the papilla. Indeed, micropuncture studies have demonstrated that in rats, the increased osmolality at the end of the $\mathrm{dtL}$ is due to water extraction by $60 \%$; the remaining $40 \%$ depend on urea reabsorption [31]. Together with electrolytes, $\mathrm{NH}_{3} / \mathrm{NH}_{4}{ }^{+}$and urea also contribute to the hyperosmolality of the interstitium. Subsequent studies have demonstrated that urea permeability is very high in the deepest sites of dtL segments lacking AQP1 [32], and that it is mediated by UTA2, contributing to the concentration process. Urea seems to be the main determinant of interstitial osmolality in the inner medulla [33]. By being reabsorbed along the $\mathrm{CD}$ through the facilitating urea trans- 
Fig. 2. Action of vasopressin $(\mathrm{ADH})$ via $\mathrm{V} 2$ receptor $(\mathrm{V} 2 \mathrm{R})$ on the principal cells. AC, adenyl cyclase; AQP2, aquaporin 2; ATP, adenosine triphosphate; cAMP, cyclic adenosine monophosphate; PKA, protein kinase $\mathrm{A}$.

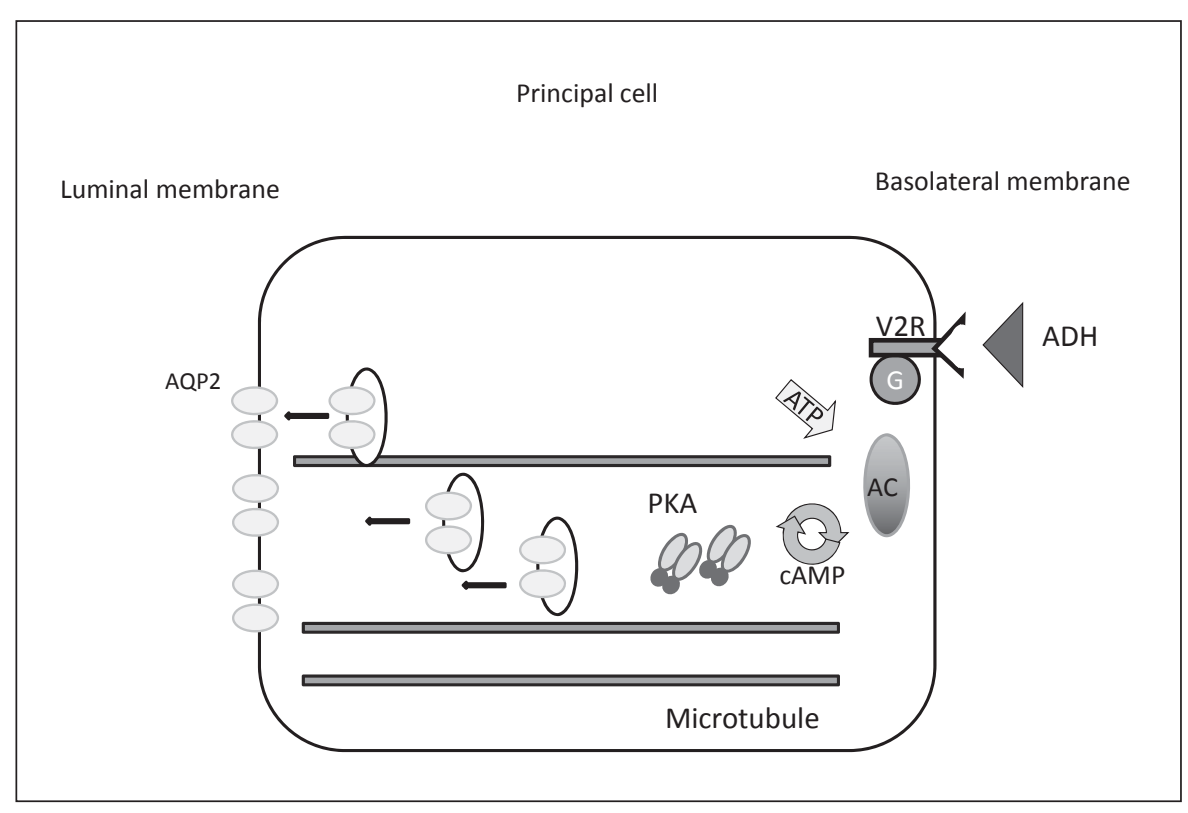

porters UTA1 and UTA3, urea accumulates in the medulla. As a toxic substance, final reabsorption in the $C D$ seems to be nonsense. However, facilitating the transport of urea via the UTA transporter family avoids urea-driven osmotic diuresis, as demonstrated in UTA1-3 knockout mice when challenged with high-protein diets [34].

The second component of the urine concentrating mechanism is the CD. CD permeability to water is continuously modulated according to the body's water needs. Vasopressin $(\mathrm{ADH})$ promotes $\mathrm{CD}$ permeability in response to several stimuli, mainly when serum osmolality tends to increase or blood volume to decrease [35]. ADH binds to the V2 receptor (V2R) on the basolateral membrane of the principal cells along the $\mathrm{CD}$. Upon $\mathrm{ADH}$ binding, V2R stimulates a cascade of events, mainly cyclic AMP-dependent ones, leading to the phosphorylation of AQP2, the water channel exclusively expressed by the principal cells of the CD. Phosphorylation at canonical serine sites of the C-terminal tail of the protein allows AQP2 trafficking towards the apical membrane of the principal cells [36] (Fig. 2). Whereas apical reabsorption is granted by AQP2, basolateral uptake of water occurs via the AQP3 and AQP4 channels. In a state of water restriction, plasmatic $\mathrm{ADH}$ levels increase, leading to AQP2 phosphorylation, actin depolymerization, and cytoskeleton rearrangement, favoring AQP2 trafficking to the apical membrane. Conversely, after large water ingestion, $\mathrm{ADH}$ release is suppressed and this mechanism arrested. Experimental studies have demonstrated that acute water

The Kidney in Bardet-Biedl Syndrome ingestion results in either renal medullary hypotonicity as well as reduced AQP2 abundance along the apical membrane of the CD. The reduced abundance of AQP2 is only in part due to reduced $\mathrm{ADH}$ plasma levels; it is possible that medullary hypotonicity alters AQP2 stability, leading to protein degradation [37]. Interestingly, Nonoguchi et al. [38] localized, in an experimental model, the V2R at both the apical and the basolateral site of the inner medullary collecting duct. Accordingly, Sarmiento et al. [39] demonstrated that in the rat kidney, V2R is mainly expressed at the luminal site during prenatal life; afterwards it is present at the basolateral and the luminal site in newborn rats, and in adult rats the basolateral expression dominates over the apical site. It has been proposed that under physiologic conditions, luminal ADH may serve as a negative feedback system for the plasmatic/basolateral action of $\mathrm{ADH}$. More recently, the primary cilium of the renal epithelium has been shown to display V2R staining in a cell culture model [40]. Its role remains to be elucidated.

\section{Pathogenesis of Urine Concentrating Defect in BBS} and Other Ciliopathies: The Evidence of Renal Origin

Altered renal water handling has been described in several ciliopathies, with a broad range of clinical consequence, from overt polyuria to the inability to maximize urine dilution and/or concentration.

As cilia are present on almost all cell types, a defect in concentrating urine may be the result of several dysfunc-

Kidney Dis 2017;3:57-65 DOI: $10.1159 / 000475500$ 
tions: (1) defective osmoregulation, leading to impaired $\mathrm{ADH}$ secretion, (2) loss of renal corticomedullary osmotic gradient due to defective salt transport or altered anatomical architecture of the medullary tubules and/or vessels, or (3) renal resistance to $\mathrm{ADH}$, due to a defective response of the $\mathrm{CD}$, for either receptorial or postreceptorial dysfunctions.

Multiple independent studies have shown that ADPKD patients with normal GFR fail to maximally concentrate urine after water restriction and infusion of desmopressin, an analog of $\mathrm{ADH}$, suggesting a renal origin of the defect $[41,42]$. This evidence raised the hypothesis that before GFR decline, for some reason polycystin dysfunction caused renal inability to correctly respond to $\mathrm{ADH}$, with the consequent defective capacity to maximally concentrate the urine. In accordance with this evidence, plasma ADH levels in ADPKD patients have been found to be higher than those of normal subjects, consistent with a compensatory increase due to kidney resistance. More recently, increased circulating copeptin levels have been described, reinforcing the idea of renal resistance and compensatory increased $\mathrm{ADH}$ plasma release [43]. These adaptations were found before the decline of the GFR, suggesting that the inability to concentrate the urine occurs at initial stages of cystic disease. The presence of high levels of $\mathrm{ADH} /$ copeptin is compatible with normal osmoregulation in ADPKD patients. However, the role of osmoregulation in ADPKD patients is still debated, given the presence of other studies showing opposite results. Bichet [44] showed that ADPKD patients had a blunted response to dehydration, demonstrating both blunted increased uOsm and plasma ADH levels. These authors suggested that beside renal resistance, defective osmoregulation may also play a role in impaired urine concentration in this setting. Studies on Pkd1+/- mice indicate that PKD1 haploinsufficiency, in the absence of renal insufficiency and cyst development, caused a blunted aquaretic response to water loading and V2R antagonists, suggesting a defective diluting efficiency. However, V2R distribution and affinity were normal, indicating that this defect was not the result of V2R dysfunction. Conversely, the urine concentrating ability after prolonged water restriction was even higher than in wildtype mice, and the AQP2 mRNA and protein abundance on the apical membrane of the $\mathrm{CD}$ were even increased [45]. Even though this model indicates the presence of impaired water handling in the case of PKD1 deficiency, it does not resemble the urine concentrating inability of ADPKD patients; however, it is hard to reach an interpretation and conclusions. So, even if impaired urine con- centration is without a doubt a common finding in ADPKD patients, the molecular basis of this dysfunction and its prognostic meaning are still obscure.

Nephronophthisis is another ciliopathy presenting with urine concentrating defect, chronic tubulointerstitial nephritis, cystic disease, and renal failure. Polyuria is generally an early sign of the disease, preceding the GFR decline. However, the pathogenesis of this defect is only partially understood. The protein products of NPHP genes, nephrocystins, have been shown to have multiple functions, including cell polarity, the formation of cellcell adherent junction, and focal adhesion [41]. It is possible that their dysfunction may lead to defective cell-cell signaling and epithelium integrity, resulting in tubulointerstitial fibrosis and dissipation of the corticomedullary osmotic gradient. However, deregulation of AQP2 trafficking due to loss of microtubular organization cannot be excluded. The main cause of polyuria as the presenting symptom has never been fully elucidated.

Harnett et al. [26] in 1988 demonstrated that BBS patients failed to reach maximum uOsm, even in the absence of a significant difference in plasma osmolality. Interestingly, uOsm did not increase significantly after desmopressin administration, indicating renal resistance to $\mathrm{ADH}$. We observed a high incidence of urine concentrating defect in BBS patients even in the absence of renal insufficiency [7]. Eight BBS patients underwent desmopressin infusion after an acute water load in order to address the renal ability to concentrate urine when endogenous $\mathrm{ADH}$ is suppressed. The study demonstrated that BBS patients were unable to significantly increase uOsm after drug administration, indicating a renal origin. Interestingly, the majority of hyposthenuric BBS patients also showed a defect in diluting the urine after an acute water load, suggesting a more complex defective water handling.

Studies Supporting a Putative Role of CD Dysfunction in the Pathogenesis of Impaired Water Handling in

BBS Patients

Only few studies have addressed the role of the CD in the pathogenesis of urine concentrating defect in BBS. Marion et al. [46] were the first to address the role of BBS proteins in water reabsorption along the $\mathrm{CD}$. They found that silencing BBS10 in human CD cell line resulted in the loss of the primary cilium, and consequently in the abrogation of V2R ciliary targeting.

The role of apical V2R remains unclear, as described above; however, this study demonstrated that BBS10 in the $\mathrm{CD}$ cells is required to detect luminal $\mathrm{ADH}$ and to
62

Kidney Dis 2017;3:57-65 DOI: $10.1159 / 000475500$
Zacchia/Di Iorio/Trepiccione/Caterino/ Capasso 
target AQP2 to the apical membrane. This fascinating explanation of the putative mechanism underlying the urine concentrating defect in BBS patients remains, however, a hypothesis to be confirmed by in vivo studies and possibly clinical investigations. We recently investigated the function of the CD in hyposthenuric BBS patients. We addressed whether renal resistance to desmopressin was based on a receptorial or postreceptorial mechanism in hyposthenuric BBS patients. For this purpose, we analyzed the time course of von Willebrand factor and factor VIII plasma levels after desmopressin infusion. The rationale underlying this study is the evidence that V2R, in endothelial cells, mediates plasma release of those coagulation factors in normal subjects. This effect is in fact blunted in patients with inactivating mutations of V2R, namely in patients with X-linked diabetes insipidus [47]. In our study, the response of the coagulation factors to desmopressin infusion resembled that of normal subjects, demonstrating that at least in endothelial cells, V2R was able to perfectly detect extracellular ADH [7]. This observation suggests a normal function of V2R; if there is any deregulation of AQP2, it is presumably located downstream of V2R. However, it is clear that V2R may have different regulation in different tissues; thus, we cannot exclude that kidney-specific V2R dysfunction may occur in BBS patients.

Urinary AQP2 excretion is considered an estimation of its abundance on the apical membrane of principal cells [48]. At baseline, after over $12 \mathrm{~h}$ of water deprivation, hyposthenuric BBS patients and age-matched healthy volunteers showed no different urinary AQP2 excretion [7]. This finding suggests that after prolonged water restriction, the lowest level of $\mathrm{uOsm}$ in BBS patients is not the consequence of impaired AQP2 trafficking. However, after acute stimuli, namely acute water loading and desmopressin infusion, patients with combined concentrating and diluting defect showed a blunted response of urinary AQP2 excretion. These findings indicate a more complex defective water handling, with impaired trafficking of AQP2 upon acute stimuli. The molecular basis linking BBS proteins and AQP2 are largely unknown.

We described a series of 4 BBS patients, where 1 patient carrying a biallelic mutation of $B B S 10$ presented with defective urine concentrating ability, while $3 \mathrm{BBS} 1$ patients did not [49]. We demonstrated that silencing either $B B S 10$ or $B B S 1$ in MCD4 cells resulted in the absence of any defect in cell polarity, but reduced abundance of ciliated cells. Intriguingly, knocking down BBS10, but not $B B S 1$, dramatically reduced AQP2 accumulation at the apical plasma membrane after forskolin stimulation.

The Kidney in Bardet-Biedl Syndrome
Confocal immunofluorescence images revealed that AQP2 was misdirected to the basolateral membrane in BBS10-silenced cells. Only BBS10-silenced cells showed a dramatic reduction in acetylated tubulin in subapical sections. As tubulin posttranslational modifications seem to provide navigational cues for molecular delivery to subcellular domains, it is possible that the reduced acetylation of tubulin may underlie the AQP2 misrouting. In conclusion, the mechanism underlying renal resistance to $\mathrm{ADH}$ has received attention in recent years; independent studies demonstrated that in vitro BBS10 knockdown caused AQP2 mistrafficking; accordingly, urinary AQP2 excretion was impaired in hyposthenuric BBS patients, corroborating the putative role of AQP2 trafficking in impaired water handling. The question whether receptorial or postreceptorial mechanism are involved requires further investigations.

Studies Supporting a Putative Role of TAL Dysfunction in the Pathogenesis of Impaired Water Handling in BBS Patients

The evidence that after prolonged water restriction there is no difference in urinary AQP2 excretion between hyposthenuric BBS patients and controls prompted us to investigate the role of other tubular segments.

The TAL is a crucial segment of the nephron in both concentrating and diluting the urine, and the majority of hyposthenuric BBS patients in our study showed a combined defect. A dysfunction of the TAL could explain both defects. Interestingly, at baseline no typical electrolyte imbalance suggesting TAL dysfunction have been ever described in BBS patients. However, it cannot be excluded that latent TAL defects unmasked by stress conditions may be present in this setting. A furosemide test could answer this question. Given the analogies between BBS and uromodulin-associated kidney disease (UAKD), including renal hyposthenuria, interstitial fibrosis, and $\mathrm{CKD}$, we hypothesized that UMOD trafficking may be affected in BBS patients. In UAKD patients, genetic mutations of UMOD cause protein maturation defects with endoplasmic reticulum retention [50, 51]. Intracellular UMOD retention is considered a key event in kidney pathology in UAKD, leading to toxic effects on the tubular cells. In accordance with intracellular accumulation, UMOD urine excretion has been found to be lower than normal in UAKD patients. We hypothesized that BBS dysfunction could alter UMOD trafficking, leading to intracellular UMOD accumulation, with subsequent TAL dysfunction. Reduced urinary UMOD excretion could be a marker of this defect. We therefore measured urinary

Kidney Dis 2017;3:57-65 DOI: $10.1159 / 000475500$ 
UMOD excretion in hyposthenuric BBS patients, demonstrating that it is reduced compared with controls. In addition, this effect was restricted to hyposthenuric BBS patients, as 3 nonhyposthenuric BBS patients showed no difference in UMOD excretion compared with controls [7]. This result is intriguing, however further studies are needed to confirm that TAL dysfunction is involved in urine concentrating defect in BBS patients. An altered architecture of medullary nephrons and/or vessels still remains another unexplored possibility.

\section{Conclusion}

Renal dysfunction in BBS patients is highly variable. Patients with severe antenatal kidney abnormalities, representing a fraction of BBS patients, reach end-stage renal disease early in life. Adult BBS patients show a wide range of kidney dysfunction. Urine concentrating de- fect, in the absence of kidney insufficiency, is a common finding. Whether this defect is an initial sign preceding a progressive decline of renal function remains to be elucidated. The mechanism underlying this defect may be the result of multiple factors. Some studies indicate tubular dysfunction as one of the putative mechanisms, with the implication of AQP2 mistrafficking. Whether additional mechanisms underlie this defect remains to be elucidated.

\section{Acknowledgments}

The authors are grateful to the Italian Society of Bardet-Biedl (ASBBI) for its support.

\section{Conflict of Interest Statement}

The authors declare no conflicts of interest.

\section{References}

1 Forsythe E, Beales PL: Bardet-Biedl syndrome. Eur J Hum Genet 2013;21:8-13.

2 Bardet G: On congenital obesity syndrome with polydactyly and retinitis pigmentosa (a contribution to the study of clinical forms of hypophyseal obesity). 1920. Obes Res 1995;3: 387-399.

3 Biedl A: A pair of siblings with adiposo-genital dystrophy. 1922. Obes Res 1995;3:404.

4 Beales PL, Elcioglu N, Woolf AS, Parker D, Flinter FA: New criteria for improved diagnosis of Bardet-Biedl syndrome: results of a population survey. J Med Genet 1999;36:437-446.

5 Castro-Sánchez S, Álvarez-Satta M, Valverde $\mathrm{D}$ : Bardet-Biedl syndrome: a rare genetic disease. J Pediatr Genet 2013;2:77-83.

6 Moore SJ, Green JS, Fan Y, Bhogal AK, Dicks E, Fernandez BA, Stefanelli M, Murphy C, Cramer BC, Dean JC, Beales PL, Katsanis N, Bassett AS, Davidson WS, Parfrey PS: Clinical and genetic epidemiology of Bardet-Biedl syndrome in Newfoundland: a 22-year prospective, population-based, cohort study. Am J Med Genet A 2005;132A:352-360.

7 Zacchia M, Zacchia E, Zona E, Capolongo G, Raiola I, Rinaldi L, Trepiccione F, Ingrosso D, Perna A, Di Iorio V, Simonelli F, Moe OW, Capasso G: Renal phenotype in Bardet-Biedl syndrome: a combined defect of urinary concentration and dilution is associated with defective urinary AQP2 and UMOD excretion. Am J Physiol Renal Physiol 2016;311:F686-F694.

8 Fulton AB, Hansen RM, Glynn RJ: Natural course of visual functions in the Bardet-Biedl syndrome. Arch Ophthalmol 1993;111:15001506.
9 Heon E, Kim G, Qin S, Garrison JE, Tavares E, Vincent A, Nuangchamnong N, Scott CA, Slusarski DC, Sheffield VC: Mutations in C8ORF37 cause Bardet Biedl syndrome (BBS21). Hum Mol Genet 2016;25:22832294.

10 Marra AN, Li Y, Wingert RA: Antennas of organ morphogenesis: the roles of cilia in vertebrate kidney development. Genesis 2016;54: 457-469.

11 Ansley SJ, Badano JL, Blacque OE, Hill J, Hoskins BE, Leitch CC, Kim JC, Ross AJ, Eichers ER, Teslovich TM, Mah AK, Johnsen RC, Cavender JC, Lewis RA, Leroux MR, Beales PL, Katsanis N: Basal body dysfunction is a likely cause of pleiotropic Bardet-Biedl syndrome. Nature 2003;425:628-633.

12 Novas R, Cardenas-Rodriguez M, Irigoín F, Badano JL: Bardet-Biedl syndrome: is it only cilia dysfunction? FEBS Lett 2015;589:34793491.

13 Mourão A, Christensen ST, Lorentzen E: The intraflagellar transport machinery in ciliary signaling. Curr Opin Struct Biol 2016;41:98108.

14 Wei Q, Zhang Y, Li Y, Zhang Q, Ling K, Hu J: The BBSome controls IFT assembly and turnaround in cilia. Nat Cell Biol 2012;14:950957.

15 Jiang J, Promchan K, Jiang H, Awasthi P, Marshall H, Harned A, Natarajan V: Depletion of BBS protein LZTFL1 affects growth and causes retinal degeneration in mice. J Genet Genomics 2016;43:381-391.
16 Su X, Driscoll K, Yao G, Raed A, Wu M, Beales PL, Zhou J: Bardet-Biedl syndrome proteins 1 and 3 regulate the ciliary trafficking of polycystic kidney disease 1 protein. Hum Mol Genet 2014;23:5441-5451.

17 Berbari NF, Lewis JS, Bishop GA, Askwith CC, Mykytyn K: Bardet-Biedl syndrome proteins are required for the localization of $\mathrm{G}$ protein-coupled receptors to primary cilia. Proc Natl Acad Sci USA 2008; 105:4242-4246.

18 Schou KB, Pedersen LB, Christensen ST: Ins and outs of GPCR signaling in primary cilia. EMBO Rep 2015;16:1099-1113.

19 Daniels AB, Sandberg MA, Chen J, Weigel-Di Franco C, Fielding Hejtmancic J, Berson EL: Genotype-phenotype correlations in BardetBiedl syndrome. Arch Ophthalmol 2012;130: 901-907.

20 Esposito G, Testa F, Zacchia M, Crispo AA, Di Iorio V, Capolongo G, Rinaldi L, D’Antonio M, Fioretti T, Iadicicco P, Rossi S, Franzè A, Marciano E, Capasso G, Simonelli F, Salvatore F: Genetic characterization of Italian patients with Bardet-Biedl syndrome and correlation to ocular, renal and audio-vestibular phenotype: identification of eleven novel pathogenic sequence variants. BMC Med Genet 2017;18:10.

21 Forsythe E, Sparks K, Best S, Borrows S, Hoskins B, Sabir A, Barrett T, Williams D, Mohammed S, Goldsmith D, Milford DV, Bockenhauer D, Foggensteiner L, Beales PL: Risk factors for severe renal disease in BardetBiedl syndrome. J Am Soc Nephrol 2017;28: 963-970. 
22 Gourdol O, David L, Colon S, Bouvier R, Ayral A, Aguercif M, François R: Renal involvement in the Laurence-Moon-Bardet-Biedl syndrome. Apropos of 3 cases (in French). Pediatrie 1984;39:175-181.

23 Tieder M, Levy M, Gubler MC, Gagnadoux MF, Broyer M: Renal abnormalities in the Bardet-Biedl syndrome. Int J Pediatr Nephrol 1982;3:199-203.

24 Churchill DN, McManamon P, Hurley RM: Renal disease - a sixth cardinal feature of the Laurence-Moon-Biedl syndrome. Clin Nephrol 1981;16:151-154.

25 O’Dea D, Parfrey PS, Harnett JD, Hefferton D, Cramer BC, Green J: The importance of renal impairment in the natural history of Bardet-Biedl syndrome. Am J Kidney Dis 1996;27:776-783.

26 Harnett JD, Green JS, Cramer BC, Johnson G, Chafe L, McManamon P, Farid NR, PrysePhillips W, Parfrey PS: The spectrum of renal disease in Laurence-Moon-Biedl syndrome. N Engl J Med 1988;319:615-618.

27 Sands JM, Layton HE: The physiology of urinary concentration: an update. Semin Nephrol 2009;29:178-195.

28 Mount DB: Thick ascending limb of the loop of Henle. Clin J Am Soc Nephrol 2014;9: 1974-1986.

29 Bockenhauer D, Bichet D: Inherited secondary nephrogenic diabetes insipidus: concentrating on humans. Am J Physiol Renal Physiol 2013;304:F1037-F1042.

30 Layton AT: Modeling transport and flow regulatory mechanisms of the kidney. ISRN Biomath 2012;2012:170594.

31 Jamison RL, Buerkert J, Lacy FB: A micropuncture study of Henle's thin loop in Brattleboro rats. Am J Physiol 1973;224:180-185.

32 Nawata CM, Evans KK, Dantzler WH, Pannabecker TL: Transepithelial water and urea permeabilities of isolated perfused MunichWistar rat inner medullary thin limbs of Henle's loop. Am J Physiol Renal Physiol 2014; 306:F123-F129.

33 Sands JM: Renal urea transporters. Curr Opin Nephrol Hypertens 2004;13:525-532.
34 Fenton RA, Flynn A, Shodeinde A, Smith CP, Schnermann J, Knepper MA: Renal phenotype of UT-A urea transporter knockout mice. J Am Soc Nephrol 2005;16:1583-1592.

35 Pearce D, Soundararajan R, Trimpert C, Kashlan OB, Deen PM, Kohan DE: Collecting duct principal cell transport processes and their regulation. Clin J Am Soc Nephrol 2015; 10:135-146.

36 Jung HJ, Kwon TH: Molecular mechanisms regulating aquaporin-2 in kidney collecting duct. Am J Physiol Renal Physiol 2016; 311:F1318-F1328.

37 Soodvilai S, Jia Z, Wang MH, Dong Z, Yang T: mPGES-1 deletion impairs diuretic response to acute water loading. Am J Physiol Renal Physiol 2009;296:F1129-F1135.

38 Nonoguchi H, Owada A, Kobayashi N, Takayama M, Terada Y, Koike J, Ujiie K, Marumo F, Sakai T, Tomita K: Immunohistochemical localization of V2 vasopressin receptor along the nephron and functional role of luminal V2 receptor in terminal inner medullary collecting ducts. J Clin Invest 1995;96: 1768-1778.

39 Sarmiento JM, Ehrenfeld P, Añazco CC, Reyes CE, Troncoso S, Figueroa CD, MüllerEsterl W, González CB: Differential distribution of the vasopressin $\mathrm{V}$ receptor along the rat nephron during renal ontogeny and maturation. Kidney Int 2005;68:487-496.

40 Raychowdhury MK, Ramos AJ, Zhang P, McLaughin M, Dai XQ, Chen XZ, Montalbetti N, Del Rocío Cantero M, Ausiello DA, Cantiello HF: Vasopressin receptor-mediated functional signaling pathway in primary cilia of renal epithelial cells. Am J Physiol Renal Physiol 2009;296:F87-F97.

41 Krishnan R, Eley L, Sayer JA: Urinary concentration defects and mechanisms underlying nephronophthisis. Kidney Blood Press Res 2008;31:152-162.

42 Martinez-Maldonado M, Yium JJ, Eknoyan G, Suki WN: Adult polycystic kidney disease: studies of the defect in urine concentration. Kidney Int 1972;2:107-113.

43 Zittema D, Boertien WE, van Beek AP, Dullaart RP, Franssen CF, de Jong PE, Meijer E, Gansevoort RT: Vasopressin, copeptin, and renal concentrating capacity in patients with autosomal dominant polycystic kidney disease without renal impairment. Clin J Am Soc Nephrol 2012;7:906-913.
44 Bichet DG: A defect in vasopressin secretion in autosomal dominant polycystic kidney disease. Kidney Int 2012;82:1051-1053.

45 Ahrabi AK, Terryn S, Valenti G, Caron N, Serradeil-Le Gal C, Raufaste D, Nielsen S, Horie S, Verbavatz JM, Devuyst O: PKD1 haploinsufficiency causes a syndrome of inappropriate antidiuresis in mice. J Am Soc Nephrol 2007; 18:1740-1753.

46 Marion V, Schlicht D, Mockel A, Caillard S, Imhoff O, Stoetzel C, van Dijk P, Brandt C, Moulin B, Dollfus H: Bardet-Biedl syndrome highlights the major role of the primary cilium in efficient water reabsorption. Kidney Int 2011;79:1013-1025.

47 Bichet DG, Razi M, Lonergan M, Arthus MF, Papukna V, Kortas C, Barjon JN: Hemodynamic and coagulation responses to 1-desamino(8-D-arginine) vasopressin in patients with congenital nephrogenic diabetes insipidus. N Engl J Med 1988;318:881-887.

48 Elliot S, Goldsmith P, Knepper M, Haughey $\mathrm{M}$, Olson B: Urinary excretion of aquaporin-2 in humans: a potential marker of collecting duct responsiveness to vasopressin. J Am Soc Nephrol 1996;7:403-409.

49 Zacchia M, Esposito G, Carmosino M, Barbieri C, Zacchia E, Crispo AA, Fioretti T, Trepiccione F, Di Iorio V, Simonelli F, Salvatore F, Capasso G, Svelto M, Procino G: Knockdown of the BBS10 gene product affects apical targeting of AQP2 in renal cells: a possible explanation for the polyuria associated with Bardet-Biedl syndrome. J Genet Syndr Gene Ther 2014;5:222.

50 Bernascone I, Vavassori S, Di Pentima A, Santambrogio S, Lamorte G, Amoroso A, Scolari F, Ghiggeri GM, Casari G, Polishchuk R, Rampoldi L: Defective intracellular trafficking of uromodulin mutant isoforms. Traffic 2006;7:1567-1579.

51 Zacchia M, Capasso G: The importance of uromodulin as regulator of salt reabsorption along the thick ascending limb. Nephrol Dial Transplant 2015;30:158-160. 